\title{
Sutureless Laparoscopic Inguinal Hernia Repair in Children - Don't Risk Injury with Sutures
}

\author{
William Kagetsu Bukowski ${ }^{1}$, Timothy Paul Bukowski ${ }^{2, ~ *}$ \\ ${ }^{1}$ Department of Biology, Georgetown University, Washington, The United States \\ ${ }^{2}$ Department of Urology, University of North Carolina at Chapel Hill, Chapel Hill, The United States \\ Email address: \\ WB415@georgetown.edu (W. K. Bukowski), Timothy_Bukowski@med.unc.edu (T. P. Bukowski) \\ ${ }^{*}$ Corresponding author
}

\section{To cite this article:}

William Kagetsu Bukowski, Timothy Paul Bukowski. Sutureless Laparoscopic Inguinal Hernia Repair in Children - Don't Risk Injury with Sutures. International Journal of Clinical Urology. Vol. 4, No. 2, 2020, pp. 73-76. doi: 10.11648/j.ijcu.20200402.19

Received: September 22, 2020; Accepted: October 6, 2020; Published: October 13, 2020

\begin{abstract}
Introduction: There have been multiple descriptions for the laparoscopic repair of inguinal hernia in children. Except for one, they all involve a suture ligation of the internal ring. Our concern is that suture ligation of the internal ring can injure the vas deferens, gonadal vessels, or cause unnecessary bleeding. We report the results of our laparoscopic inguinal hernia repairs, where we incise the peritoneum of the internal ring, and dissect the tunica vaginalis for $1 \mathrm{~cm}$. We do not suture ligate the internal ring. This includes patients who underwent laparoscopic evaluation and treatment for intraabdominal testicles, and thus have 6 month laparoscopic follow-up of those patients having a staged orchiopexy. Study design: We reviewed the charts from 2005 to 2016 of all patients with CPT codes 54692 (laparoscopic orchiopexy) and 49650 (laparoscopic inguinal hernia). During laparoscopy for the nonpalpable testicle, we proceeded to either a one stage or 2 stage orchiopexy. When we observed inguinal hernia opening on the contralateral side of the undescended testicle, we repaired it by incising around the internal ring, and carefully excising the tunica vaginalis from the internal ring for a distance of $1 \mathrm{~cm}$, leaving the internal oblique muscle to heal together, and peritoneum to resurface the area, without using sutures. (This is the same as is done for the laparoscopic orchiopexy side). In those patients with a staged orchiopexy, and we reevaluated the hernia sight for closure. Results: We reviewed charts of 87 patients with ages ranging from 3 months to 10 years. Eighteen of these had bilateral laparoscopic orchiopexy, 38 had left, and 24 had right. We performed 21 bilateral laparoscopic hernia repairs, 27 had left only, and 18 had right only. Of those patients with hernias, 11 hernias were found on the side opposite an undescended testis. In 4 patients we had the opportunity to perform a second stage orchiopexy six months later and observed the inguinal ring had closed. Follow up was at least 6 months for all patients, and no clinical hernias resulted. Conclusion: Laparoscopic inguinal hernia repair in children can be successfully and safely performed by excising the hernia sac (patent processus vaginalis) from the inguinal canal, and does not require suture ligation of the internal ring.
\end{abstract}

Keywords: Laparoscopic Inguinal Hernia, Laparoscopic Orchiopexy, Pediatric

\section{Introduction}

Inguinal hernias occur in approximately 1 out of every 100 boys. They can occur on the contralateral side in approximately $20 \%$ of boys under the age of two. An undescended testicle usually has a hernia associated with it, especially if it's an intra-abdominal testicle. During a laparoscopic orchiopexy for correction of the intra-abdominal testicle, one may find a long-looped vas that courses with a patent processus vaginalis through an open internal ring and down toward the scrotal area. The open internal ring is considered an inguinal hernia, as if left alone will become clinically apparent as the child grows and develops increasing abdominal pressure. During a standard laparoscopic orchiopexy, the peritoneum is released from the patent processus vaginalis at the internal ring. By incising the peritoneum around the internal ring, the testicle is brought down, either through the internal ring, or medial to the epigastric vessels. The peritoneum is not typically closed at the internal ring, and persistent clinical hernias are rare $[1,2]$. Occasionally during the procedure, a hernia is noted at the 
inguinal ring contralateral to that of the abdominal testicle. If the orchiopexy is done as a two stage procedure, one can fix a contralateral hernia at the first laparoscopy, and verify it is closed at the second laparoscopy 6 months later.

In a standard open pediatric inguinal hernia repair, one suture ligates the hernia sac at the internal ring, and the internal ring musculature is not reinforced. There have been many laparoscopic pediatric inguinal hernia repair procedures described, [3] and in all but two papers, they involve a suture ligation of the internal ring. [4] Our concern is that suture ligation of the internal ring can injure the vas deferens or gonadal vessels, or cause unnecessary bleeding. We describe a repair using a sutureless repair of the internal ring for pediatric inguinal hernia of the contralateral side, and we provide follow-up visual documentation of a closed internal ring in a subset of patients who have undergone a two-stage Fowler Stephens repair.

\section{Methods}

We reviewed the charts from 2005 to 2016 of all patients with CPT codes 54692 (laparoscopic orchiopexy) or 49650 (laparoscopic inguinal hernia). Patients included were those requiring a laparoscopic orchiopexy with findings of an inguinal hernia on the contralateral side. For the nonpalpable testicle, we performed either a one stage or two stage laparoscopic orchiopexy. As part of the orchiopexy, a peritoneal incision was carried around the internal ring, freeing the peritoneal tissue off the gonadal vessels and vas. When we observed an inguinal hernia opening on the contralateral side from the undescended testicle, we repaired it by incising around the internal ring, and carefully excising the tunica vaginalis from the internal ring for a distance of 1 $\mathrm{cm}$, leaving the internal oblique muscle to adhere during the healing process and to allow the peritoneum to resurface the area. Sutures were avoided with this technique. In those patients with a staged orchiopexy, we reevaluated the hernia sight for closure at the 6 month second stage procedure. All patients had follow up of at least 6 months to check for clinical evidence of hernia.

\section{Results}

We reviewed charts of 87 patients with ages ranging from 3 months to 10 years who underwent laparoscopic orchiopexy. Of these, 18 underwent bilateral laparoscopic orchiopexy, 38 had left, and 24 had right intraabdominal testicles. We performed twenty-one bilateral laparoscopic hernia repairs and 46 unilateral repairs (27 left, 18 right). Some patients with an intraabdominal testicle did not have a hernia on the affected side, and some patients with a vanished testis or laparoscopic varicocele had a hernia on the contralateral side. Of those patients with hernias, 11 hernias were found on the side opposite an undescended testis. None of our patients had an inguinal defect greater than $1 \mathrm{~cm}$. Four patients underwent a 2nd stage orchiopexy 6 months after the first stage, where we confirmed laparoscopically that the inguinal opening had closed after repair. (Figures 1-5) Follow up was at least 6 months for all patients. There were no instances of clinically significant hernias, no development of hydroceles and no testicular atrophy.

\section{Discussion}

The incidence of the pediatric inguinal is $1 \%$, and during repair, about $20-40 \%$ of patients under age two will have a patent process vaginalis on the contralateral side to the clinically apparent side. [5] About 25\% of these with contralateral patent processus vaginalis, if left alone, will become clinically apparent over time. [3, 6] Palmer described looking at the contralateral ring during laparoscopic orchiopexy, and found about a $9 \%$ incidence of hernia. [1] The hernias were repaired with a suture technique, and there was no evidence of hernia at 4 months postop. Our method of laparoscopic inguinal hernia resection involves incision around the peritoneum and careful dissection of the patent processes vaginalis from the inguinal canal for about a length of $1 \mathrm{~cm}$. We do not suture the internal ring. We have four boys with postop pictures from six months later. We had no recurrences.

Most described laparoscopic repairs of the hernia include an intra- or extraperitoneal suture technique. Recently, Davies et al for the International Pediatric Endosurgery Group reviewed the literature on the safety and efficacy of laparoscopic hernia repair, comparing it to open inguinal hernia repair, and found the for bilateral hernias, laparoscopic repair is more facile and quicker while having no increase in recurrence rate. [3] There have been a number of published papers using suture to close the internal ring, without resection of the peritoneum at the internal ring. [7-11] Some have questioned whether it is important to resect some of the peritoneum, while still using suture to close the internal ring muscle. [12] Pant compared resection of the peritoneal ring versus resection of the peritoneal ring with suture, and found that suturing made no difference unless the hernia opening was greater than $10 \mathrm{~mm}$, in which case they had three recurrences, (two in nonsuture group, and one in the suture group). [13] Riquelme found complete success during laparoscopy when he resected the tunica vaginalis from the inguinal canal and did not suture the peritoneum, unless the internal ring was greater than $10 \mathrm{~mm}$. They had no recurrences. [4] Weaver et al and Centeno-Wolf et al have determined that leaving a patent processus vaginalis opening results in a clinical problem in $10-15 \%$ of patients if left open $[14,15]$ The complications from hernia repair include atrophic testis, hydrocele formation, re-assent of the testis, and recurrence. [3] Infection rate is minimal. Most recurrence rates are somewhere between 1.4 and 3\%. The larger current rates occur in patients with larger hernia defects. In our small series of laparoscopic hernia repair, we had no recurrences, no infections, and no testis atrophy. We did not use sutures after resection of the internal ring peritoneum, but would have considered using them if the opening was greater than $10 \mathrm{~mm}$. 


\section{Conclusion}

Patent processus vaginalis, when left untreated, has an unknown true risk of developing into a clinical hernia. Using suture to close the defect is not necessary unless it is a defect over $10 \mathrm{~mm}$. Laparoscopic inguinal hernia repair in children can be successfully, and safely performed by excising the hernia sac (patent processus vaginalis) from the inguinal canal and does not require suture ligation of the internal ring. This can prevent inadvertent injury to the gonadal vessels or vas deferens during suture placement.
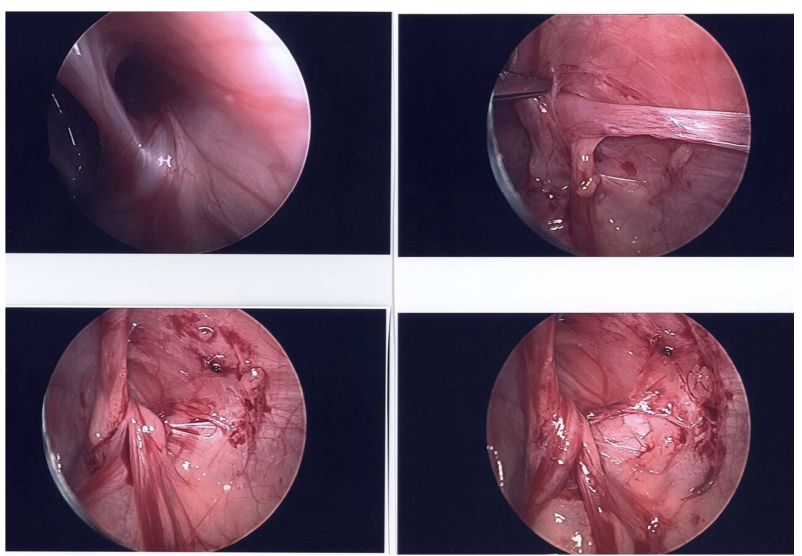

Figure 1. Excision of right inguinal processus vaginalis.

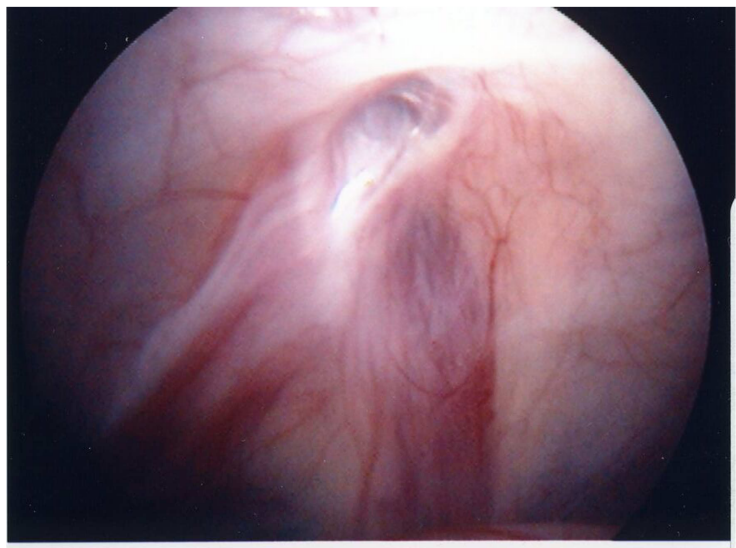

Figure 2. Same patient as above, 6 month follow up.
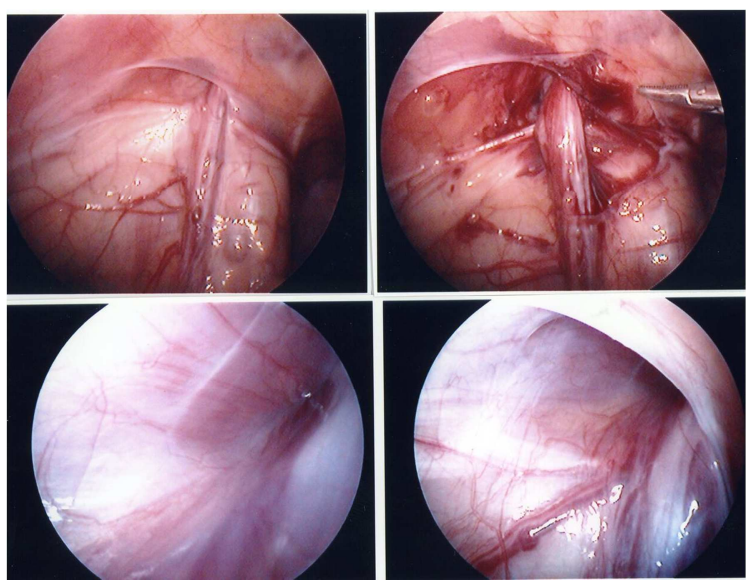

Figure 3. Laparoscopic excision of left patent processus vaginalis (top), with 6 month follow-up (bottom).

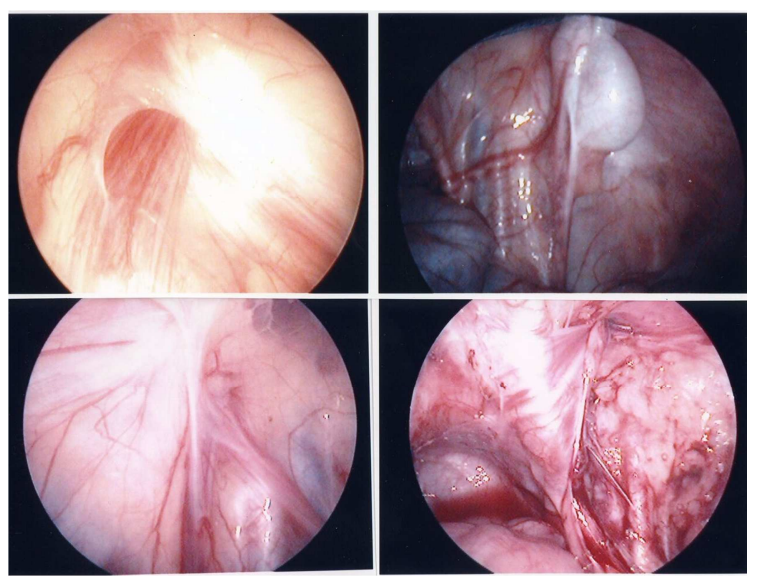

Figure 4. Laparoscopic view and left inguinal hernia and 6 month follow-up. Right side underwent staged Laparoscopic orchiopexy.
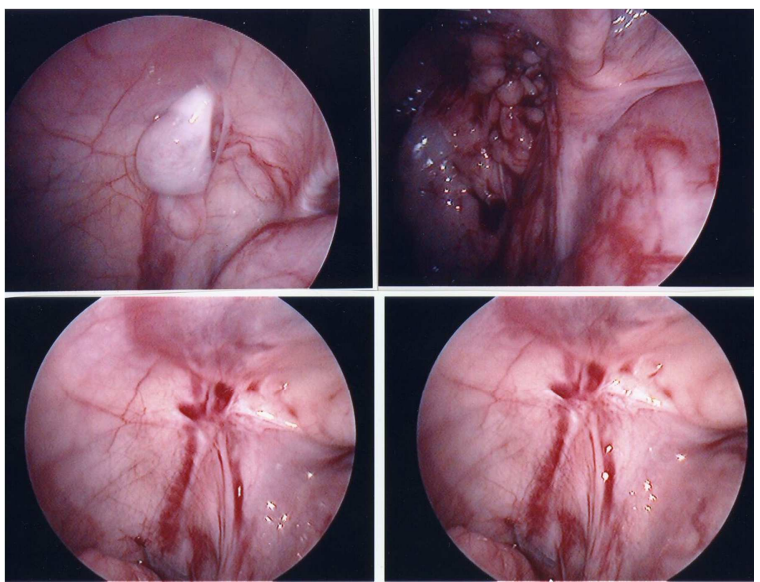

Figure 5. Left laparoscopic orchiopexy with 6 month follow-up showing closed inguinal ring.

\section{Acknowledgements}

The authors would like to thank J. Duncan Phillips, MD, Chief of Pediatric Surgery at Wake Med Hospital, Raleigh USA for his review and suggestions for the manuscript.

\section{References}

[1] Palmer, LS.; Rastinehad, A. Incidence and concurrent laparoscopic repair of intra-abdominal testis and contralateral patent processus vaginalis. Urology, August 200872 (2): 297299, http: //dx.doi.org/10.1016/j.urology.2007.12.099.

[2] Blackburn, SC; Adams, SD; and Mahomed, AA. Risk of hernia occurrence where division of an indirect inguinal sac without ligation is undertaken. Journal of Laparoendoscopic \& Advanced Surgical Techniques. September 2012, 22 (7): 713-714. doi: 10.1089/lap.2012.0011.

[3] Davies, DA.; Rideout, DA.; Clarke, SA. The International Pediatric Endosurgery Group Evidence-Based Guideline on Minimal Access Approaches to the Operative Management of Inguinal Hernia in Children. Journal of Laparoendoscopic \& Advanced Surgical Techniques. January 2017, ahead of print. doi: 10.1089/lap.2016.0453. 
[4] Riquelme, M; Aranda, A; Riquelme-Q, Mario. Laparoscopic pediatric inguinal hernia repair: no ligation, just resection. Journal of Laparoendoscopic \& Advanced Surgical Techniques. January 2010, 20 (1): 77-80. doi: 10.1089/lap.2008.0329.

[5] Tamaddon, H; Phillips, JD; Nakayama, DK. Laparoscopic evaluation of the contralateral groin in pediatric inguinal hernia patients: a comparison of 70- and 120-degree endoscopes. Journal of Laparoendoscopic \& Advanced Surgical Techniques. December 2005, 15 (6): 653-660. doi: 10.1089/lap.2005.15.653.

[6] Kokorowski PJ, wang HH, Routh JC, Hubert KC, Nelson CP. Evaluation of the contralateral inguinal ring in clinically unilateral inguinal hernia. A systematic review and metanalysis. Hernia 2014; 18: 311-324.

[7] Shalaby R, Ismail M, Dorgham A, Hefny K, Alsaied G, Gabr $\mathrm{K}$, Abelaziz M. Laparoscopic hernia repair in infancy and childhood: evaluation of 2 different techniques. J Pediatr Surg 2010 Nov; 45 (11): 2210-6.

[8] Esposito, C.; Escolino, M.; Cortese, G. et al. Twenty-year experience with laparoscopic inguinal hernia repair in infants and children: considerations and results on 1833 hernia repairs Surg Endosc (2017) 31: 1461. doi: 10.1007/s00464-016-51398.

[9] Grimsby, GM.; Keays, MA.; Villanueva, C.; Bush, NC.; Snodgrass, WT.; Gargollo, PC.; Jacobs, MA. Non-absorbable sutures are associated with lower recurrence rates in laparoscopic percutaneous inguinal hernia ligation. $J$ Pediatr Urol. 2015 Oct; 11 (5): 275. doi: 10.1016/j.jpurol.2015.04.029. Epub 2015 Jun 14.
[10] McClain, L., Streck, C., Lesher, A. et al. Laparoscopic needleassisted inguinal hernia repair in 495 children. Surg Endosc (2015) 29: 781. doi: 10.1007/s00464-014-3739-8 .

[11] Thomas, DT.; Göcmen, KB.; Tulgar, S.; Boga, I. Percutaneous internal ring suturing is a safe and effective method for the minimal invasive treatment of pediatric inguinal hernia: Experience with 250 cases. J Pediatr Surg. 2016 Aug; 51 (8): 1330-5. doi: 10.1016/j.jpedsurg.2015.11.024. Epub 2015 Dec 11 .

[12] Borkar, NB.; Pant, N; Ratan, S; Aggarwal, SK. Laparoscopic repair of indirect inguinal hernia in children: does partial resection of the sac make any impact on outcome? Journal of Laparoendoscopic \& Advanced Surgical Techniques. March 2012, 22 (3): 290-294. doi: 10.1089/lap.2011.0259.

[13] Pant N, Aggarwal SK, Ratan SK. Laparoscopic repair of hernia in children: Comparison between ligation and nonligation of sac. J Indian Assoc Pediatr Surg 2014; 19: 76-9. doi: 10.4103/0971-9261.129597.

[14] Weaver KL, Poola AS, Gould JL, Sharp SW, St Peter SD, Holcomb GW 3rd. The risk of developing a symptomatic inguinal hernia in children with an asymptomatic patent processus vaginalis. J Pediatr Surg. 2017 Jan; 52 (1): 60-64. doi: 10.1016/j.jpedsurg.2016.10.018. Epub 2016 Oct 28. PMID: 27842956.

[15] Centeno-Wolf N, Mircea L, Sanchez O, Genin B, Lironi A, Chardot C, Birraux J, Wildhaber BE. Long-term outcome of children with patent processus vaginalis incidentally diagnosed by laparoscopy. J Pediatr Surg. 2015 Nov; 50 (11): 1898-902. doi: 10.1016/j.jpedsurg.2015.07.001. Epub 2015 Jul 4.PMID: 6233492. 